\title{
A new necessary and sufficient condition for the simultaneous stabilization of three time-varying linear systems
}

\author{
Liu Liu*, Lu Yufeng \\ School of Mathematical Sciences, Dalian University of Technology, Dalian 116024 China \\ *Corresponding author, e-mail: beth.liu@dlut.edu.cn
}

Received 4 May 2018

Accepted 26 Apr 2019

\begin{abstract}
This paper considers the simultaneous stabilization problems of three discrete-time time-varying linear systems within the framework of nest algebra. Under the transmission condition, a necessary and sufficient condition for the existence of simultaneously stabilizing controllers is given based on the strong representation. Some simultaneous stabilizability criteria are established in terms of single strong representation of only one controller in the transmission condition.
\end{abstract}

KEYWORDS: simultaneous stabilization, transitivity, nest algebra, strong representation, time-varying system

MSC2010: 47L35 47N70

\section{INTRODUCTION}

The stability for different types of discrete-time time-varying linear systems are studied in Refs. 1-4. The control theory of time-varying linear systems in the framework of nest algebra, which was sparked and developed based on the nest algebra theory in 1980s, is a generalization of $H^{\infty}$ control in the sense that 2-norm is used to quantify the size of signals. In the context of operator theory, the timevarying linear system can be seen as a (possibly unbounded) infinite-dimensional lower triangular matrix. The stability of the system is equivalent to the boundedness of the infinite-dimensional matrix. This approach is purely operator theoretic and does not use any state space realization. This interpretation of dynamical systems has generated significant research interest over the last decade, as witnessed by recent contributions ${ }^{5-7}$.

The simultaneous stabilization problem concerns the design of a common controller that would stabilize a finite set of plants, and it was first introduced by Saeks et $\mathrm{al}^{8}$ and Viswanadham ${ }^{9}$. The simultaneous stabilization problem for three systems is recognized as one of hard open problems in linear system theory. Few criteria for simultaneous stabilizability appear without any restrictions to the given plants. The traditional necessary and sufficient conditions for simultaneous stabilization of time-varying linear systems are all characterized in the form of strong representations of all given plants ${ }^{10,11}$. However, the problem of how to compute strong representations of a general time-varying linear system remains unsolved. Motivated by practical interest, some new simultaneous stabilizability criteria with weaker condition about strong representations of the plants are needed. In 2011, Yu and Han brought out the socalled transitivity in simultaneous stabilization for three time-varying linear systems, which, when satisfied, will lead to characterizations of simultaneous stabilizability and simultaneously stabilizing feedback controllers ${ }^{12}$. In other words, the transitivity problem restricts the search for the existence of a simultaneously stabilizing controller to the three plants satisfying the so-called transmission condition. The transmission condition for three given plants $L_{0}, L_{1}, L_{2}$ is that $L_{0}, L_{1}$ can be simultaneously stabilized by some system $C_{0}$, and $L_{1}, L_{2}$ can be simultaneously stabilized by some system $C_{1}$. It was pointed out that the transmission condition really is a weaker replacement of the strong representation condition in the study of simultaneous stabilization problem ${ }^{13}$. Under the transmission condition, a necessary and sufficient condition for the simultaneous stabilizability of three plants $L_{0}, L_{1}$, and $L_{2}$ was given, and the strong representation of $L_{2}$ was avoided $^{13}$. Some sufficient conditions for the simultaneous stabilizability are given in Ref. 14-those 
conditions do not employ strong representations of $L_{0}$ and $L_{2}$. As an improvement of the previous work, an equivalent condition not depending on the strong representations of $L_{0}$ or $L_{2}$ will be derived ${ }^{15}$. Up to the present, some criteria for simultaneous stabilizability of three plants have been given within framework of nest algebra. While, those results were all established in terms of strong representations of at least one plant, it is natural to ask whether the simultaneous stabilization can be characterized by strong representations of fewer given plants or fewer controllers.

The main goal of this study is to rebuild the simultaneous stabilization criteria of three plants under the transmission condition. Compared with the sufficient conditions in Refs. 12,14, one of main contributions here is establishing a necessary and sufficient condition to the transitivity of timevarying linear systems. The early equivalent conditions to the simultaneous stability depend on at least one strong representation of the considering plants and one of the controllers $7,13,15$, while the simultaneous stability criteria depend only on the single strong representation of one controller, which avoids any information about strong representations of each given plant. Additionally, a parametrization for all simultaneous stabilizers is derived under the restrictive transmission condition. In particular, a simultaneous stabilization criterion avoiding any strong representation can be established when one of the stabilizing controllers becomes stable.

\section{PRELIMINARIES}

The signal space considered in this paper is the complex separable Hilbert space

$$
\mathscr{H}=\left\{\left(x_{0}, x_{1}, \ldots\right): x_{i} \in \mathbb{C}, \sum_{i=0}^{\infty}\left|x_{i}\right|<\infty\right\},
$$

and $\mathscr{H}_{e}=\left\{\left(x_{0}, x_{1}, \ldots\right): x_{i} \in \mathbb{C}\right\}$ denotes the extended space of $\mathscr{H}$.

Let $\mathscr{B}(\mathscr{H})$ denote the Banach space of bounded linear operators on $\mathscr{H}$, endowed with the operator norm

$$
\|T\|=\sup _{x \in \mathscr{H},\|x\| \leqslant 1}\|T x\| .
$$

For $T \in \mathscr{B}(\mathscr{H}), \operatorname{Im} T$ denotes the range of $T$ and Ker $T$ denotes the kernel of $T$.

For each $n \geqslant 0$, we denote by $P_{n}$ the standard truncation projection on $\mathscr{H}$ or $\mathscr{H}_{e}$ as

$$
P_{n}\left(x_{0}, x_{1}, \ldots, x_{n}, \ldots\right)=\left(x_{0}, x_{1}, \ldots, x_{n}, 0, \ldots\right)
$$

with $P_{-1}=0$ and $P_{\infty}=I$.
$\left\{P_{n}:-1 \leqslant n \leqslant \infty\right\}$ is used to define the physical definition of causality. A linear transformation $L$ on $\mathscr{H}_{e}$ is causal if $P_{n} L P_{n}=P_{n} L$ for each $n . L$ is a (time-varying) linear system if it is a causal linear transformation on $\mathscr{H}_{e}$ and continuous with respect to the standard seminorm topology ${ }^{11}$. Indeed, any linear system is an infinite-dimensional lower triangular matrix with respect to the standard basis of $\mathscr{H}$. A linear system is stable if its restriction to $\mathscr{H}$ is a bounded operator.

Let $\mathscr{L}$ be the algebra of linear systems with respect to the standard addition and multiplication. The set of stable ones, denoted by $\mathscr{S}$, referred to in the operator theory literature as a nest algebra determined by the discrete nest ${ }^{11}\left\{I-P_{n}:-1 \leqslant\right.$ $n \leqslant \infty\} . \mathscr{S}$ is a non-self adjoint sub-algebra of the Banach algebra $\mathscr{B}(\mathscr{H})$, closed in the weak operator topology.

The definition of stabilizability and its characterization in terms of strong representations are given as follows.

Consider the feedback configuration contributed by the plant $L \in \mathscr{L}$ and the controller $C \in \mathscr{L}$, where the closed-loop equation is

$$
\left[\begin{array}{l}
u_{1} \\
u_{2}
\end{array}\right]=\left[\begin{array}{rr}
I & C \\
-L & I
\end{array}\right]\left[\begin{array}{l}
e_{1} \\
e_{2}
\end{array}\right] .
$$

The closed-loop system $\{L, C\}$ is well posed if the internal input $\left[e_{1} e_{2}\right]^{\mathrm{T}}$ can be expressed as a causal function of the external input $\left[u_{1} u_{2}\right]^{\mathrm{T}}$. This is equivalent to requiring that $\left[\begin{array}{rr}I & C \\ -L & I\end{array}\right]$ being invertible. The inverse is given by the transfer matrix

$$
H(L, C)=\left[\begin{array}{rr}
(I+C L)^{-1} & -C(I+L C)^{-1} \\
L(I+C L)^{-1} & (I+L C)^{-1}
\end{array}\right] .
$$

Definition 1 (1) The closed-loop system $\{L, C\}$ is stable if each entry of $H(L, C)$ belongs to $\mathscr{S}$.

(2) The plant $L$ is stabilizable if there exists a causal system $C \in \mathscr{L}$ such that $\{L, C\}$ is stable. In this case, $C$ is said to be a stabilizing controller for $L$.

(3) A family of plants $L_{0}, L_{1}, \ldots, L_{n}$ are simultaneously stabilizable if they admits a common stabilizing controller.

Definition 2 [Ref. 16] Let $M, N, \hat{M}, \hat{N} \in \mathscr{S}$ and $L \in$ $\mathscr{L}$.

(1) $[M N]^{\mathrm{T}}$ is a strong right representation of $L$ if $\mathscr{G}(L)=\operatorname{Im}[M N]^{\mathrm{T}}$ and there exist $X, Y \in \mathscr{S}$ such that $[Y X][M N]^{\mathrm{T}}=I$. 
(2) $[-\hat{N} \hat{M}]$ is a strong left representation of $L$ if $\mathscr{G}(L)=\operatorname{Ker}[-\hat{N} \hat{M}]$ and there exist $\hat{X}, \hat{Y} \in \mathscr{S}$ such that $[-\hat{N} \hat{M}][\hat{X} \hat{Y}]^{\mathrm{T}}=I$, where $\mathscr{G}(L)$ is the graph of $L$, i.e., $\mathscr{G}(L)=$ $\left\{[x L x]^{\mathrm{T}}: x \in \mathscr{H}, L x \in \mathscr{H}\right\}$.

Remark 1 In fact, the strong representation is an alternative, but equivalent, approach to the coprime factorization in stability theory of linear systems. More precisely, $[M N]^{\mathrm{T}}$ is a strong right representation of $L$ if and only if $L=N M^{-1}$ with $M^{-1} \in \mathscr{L}$ and $M, N$ are right-coprime. Similarly, $[-\hat{N} \hat{M}]$ is a strong left representation of $L$ if and only if $L=$ $\hat{M}^{-1} \hat{N}$ with $\hat{M}^{-1} \in \mathscr{L}$ and $\hat{M}, \hat{N}$ are left-coprime.

Let $\mathscr{A}$ be a Banach algebra containing identity and $\mathscr{A}^{n \times m}$ be the set consisting of all the $n \times m$ matrices with the elements in $\mathscr{A}$.

Definition 3 [Ref. 17] $\mathscr{A}$ is completely finite if every right invertible element $R \in \mathscr{A}^{1 \times n}$ is the first row of an invertible matrix in $\mathscr{A}^{n \times n}$ for all $n \geqslant 1$. A Banach algebra $\mathscr{A}$ is stably finite if every right (left) invertible element $A \in \mathscr{A}^{n \times n}$ is invertible in $\mathscr{A}^{n \times n}$ for all $n \geqslant 1$.

Lemma 1 (Ref. 17) If Banach algebra $\mathscr{A}$ is completely finite, then it is stably finite.

Proposition 1 (Ref. 17) The nest algebra $\mathscr{S}$ is completely finite.

The lemmas below are used to formulate the main results shown in the next section.

Lemma 2 (Ref. 15) A linear system $L \in \mathscr{L}$ is stabilizable if and only if $L$ has a strong right representation.

Lemma 3 (Ref. 11) Assume that $[M N]^{\mathrm{T}}$ is a strong right representation of $L$, and $[V U]^{\mathrm{T}}$ and $[-\hat{U} \hat{V}]$ are the strong left and right representations of $C$, respectively. Then the followings are equivalent:

(1) $\{L, C\}$ is stable.

(2) $\hat{V} M+\hat{U} N$ is invertible in $\mathscr{S}$.

(3) $\left[\begin{array}{rr}M & -U \\ N & V\end{array}\right]$ is invertible in $\mathscr{S}^{2 \times 2}$.

\section{MAIN RESULTS}

In this section, we deal with the simultaneous stabilization problem of three given plants $L_{0}, L_{1}$, and $L_{2}$ with restriction of the transmission condition. The following result is an extension of the classical Youla Parametrization Theorem, which is stated in terms of a strong right, but not both, representation.
Lemma 4 A linear system $L$ is stabilizable if and only if $L$ admits a right representation $[M N]^{\mathrm{T}}$ with $\left[\begin{array}{rr}M & -\hat{X} \\ N & \hat{Y}\end{array}\right]$ invertible in $\mathscr{S}^{2 \times 2}$ for some $\hat{X}, \hat{Y} \in \mathscr{S}$. A linear system $C \in \mathscr{L}$ stabilizes $L$ if and only if it admits a strong right representation $\left[\begin{array}{c}\hat{Y}-N Q \\ \hat{X}+M Q\end{array}\right]$ for some $Q \in \mathscr{S}$.

Proof: According to Lemma 2, $L$ is stabilizable if and only if $L$ has a strong representation $[M N]^{\mathrm{T}}$. By the complete finiteness of $\mathscr{S},[M N]^{\mathrm{T}}$ is the first column of an invertible matrix in $\mathscr{S}^{2 \times 2},\left[\begin{array}{rr}M & -\hat{X} \\ N & \hat{Y}\end{array}\right]$. Note that

$$
\left[\begin{array}{rr}
M & -(\hat{X}+M Q) \\
N & \hat{Y}-N Q
\end{array}\right]=\left[\begin{array}{rr}
M & -\hat{X} \\
N & \hat{Y}
\end{array}\right]\left[\begin{array}{rr}
I & -Q \\
0 & I
\end{array}\right] .
$$

By the stable finiteness of $\mathscr{S}$, it is clear that $\left[\begin{array}{rr}M & -(\hat{X}+M Q) \\ N & \hat{Y}-N Q\end{array}\right]$ is invertible in $\mathscr{S}^{2 \times 2}$ for every $Q \in \mathscr{S}$. Choose $Q \in \mathscr{S}$ such that $\hat{Y}-N Q$ is invertible in $\mathscr{L}$, then $\left[\begin{array}{c}\hat{Y}-N Q \\ \hat{X}+M Q\end{array}\right]$ is a strong right representation of some linear system $C \in \mathscr{L}$. According to Lemma $3, C$ is a stabilizing controller for $L$. The converse is obvious by Lemma 3 .

In Ref. 13, the authors provide a design method of simultaneously stabilizing controller for three plants $L_{0}, L_{1}$, and $L_{2}$ under the transmission condition, which is characterized in the form of strong representations of the plants $L_{0}, L_{1}$ and the controller $C_{2}$. Next, we give a simpler and useful simultaneous stabilizability criterion of three plants in terms of a strong right representation of only one controller $C_{2}$.

Theorem 1 Suppose that $C_{0}$ stabilizes $L_{0}$ and $L_{1}$ and $C_{2}$ stabilizes $L_{1}$ and $L_{2}$. Let $[V U]^{\mathrm{T}}$ be a right representation of $C_{2}$ with $\left[\begin{array}{rr}V & -\hat{X} \\ U & \hat{Y}\end{array}\right]$ invertible in $\mathscr{S}^{2 \times 2}$. Set $T_{1}=\left(L_{1} U+V\right)^{-1}\left(L_{1} \hat{Y}-\hat{X}\right)$. Then $L_{0}, L_{1}$, and $L_{2}$ are simultaneously stabilizable if and only if there exists $Q \in \mathscr{S}$ such that

$$
\begin{aligned}
\left\{L_{0} U+V\right. & \left.+\left(L_{0}-L_{1}\right)\left(I+C_{0} L_{1}\right)^{-1}\left(C_{0} V-U\right)\right\}^{-1} \\
& \times\left\{L_{0} U+V+\left(L_{0}-L_{1}\right)\left(\hat{Y}-U T_{1}\right) Q\right\}
\end{aligned}
$$

and

$$
I+\left(V+L_{2} U\right)^{-1}\left(L_{2}-L_{1}\right)\left(\hat{Y}-U T_{1}\right) Q
$$

are both invertible in $\mathscr{S}$. Moreover, $C \in \mathscr{L}$ stabilizes $L_{0}, L_{1}$, and $L_{2}$ if and only if $C$ has a strong right 
representation $\left[\begin{array}{l}V-\left(\hat{Y}-U T_{1}\right) Q \\ U+\left(\hat{X}+V T_{1}\right) Q\end{array}\right]$ for some $Q \in \mathscr{S}$ such that (1) and (2) are invertible in $\mathscr{S}$.

Proof: Define the operator

$$
T_{2}=\left(L_{2} U+V\right)^{-1}\left(L_{2} \hat{Y}-\hat{X}\right) .
$$

Suppose that $\left[-\hat{N}_{L_{j}} \hat{M}_{L_{j}}\right]$ is a strong left representation of $L_{j}, j=0,1,2$. For $i=1,2$, it follows from the stability of $\left\{L_{i}, C_{2}\right\}$ that $\hat{N}_{L_{i}} U+\hat{M}_{L_{i}} V$ is invertible in $\mathscr{S}$, hence

$$
T_{i}=\left(\hat{N}_{L_{i}} U+\hat{M}_{L_{i}} V\right)^{-1}\left(\hat{N}_{L_{i}} \hat{Y}-\hat{M}_{L_{i}} \hat{X}\right) \in \mathscr{S} .
$$

Assume that $C_{2}$ has a strong left representation $\left[-\hat{N}_{C_{2}} \hat{M}_{C_{2}}\right] . \quad$ By the complete finiteness of $\mathscr{S}$, $\left[-\hat{N}_{C_{2}} \hat{M}_{C_{2}}\right]$ can be extended to an invertible element of $\mathscr{S}^{2 \times 2}$, say $\left[\begin{array}{cc}Y & X \\ -\hat{N}_{C_{2}} & \hat{M}_{C_{2}}\end{array}\right]$. It follows from the stable finiteness of $\mathscr{S}$ that

$$
\begin{aligned}
{\left[\begin{array}{cc}
Y & X \\
-\hat{N}_{C_{2}} & \hat{M}_{C_{2}}
\end{array}\right]\left[\begin{array}{cc}
V & -\hat{X} \\
U & \hat{Y}
\end{array}\right] } & \\
& =\left[\begin{array}{cc}
Y V+X U & -Y \hat{X}+X \hat{Y} \\
0 & \hat{N}_{C_{2}} \hat{X}+\hat{M}_{C_{2}} \hat{Y}
\end{array}\right]
\end{aligned}
$$

is invertible in $\mathscr{S}^{2 \times 2}$, which implies that $\hat{N}_{C_{2}} \hat{X}+$ $\hat{M}_{C_{2}} \hat{Y}$ is invertible in $\mathscr{S}$. Then

$$
\begin{aligned}
\hat{Y}-U T_{i} & =\hat{Y}-U\left(L_{i} U+V\right)^{-1}\left(L_{i} \hat{Y}-\hat{X}\right) \\
& =\hat{Y}-C_{2}\left(L_{i} C_{2}+I\right)^{-1}\left(L_{i} \hat{Y}-\hat{X}\right) \\
& =\left(I+C_{2} L_{i}\right)^{-1}\left(\hat{Y}+C_{2} \hat{X}\right) \\
& =\left(I+C_{2} L_{i}\right)^{-1} \hat{M}_{C_{2}}^{-1}\left(\hat{N}_{C_{2}} \hat{X}+\hat{M}_{C_{2}} \hat{Y}\right)
\end{aligned}
$$

is invertible in $\mathscr{L}$. Then one can compute that

$$
L_{i}=\left(\hat{X}+V T_{i}\right)\left(\hat{Y}-U T_{i}\right)^{-1}, \quad i=1,2 .
$$

Note that

$$
\begin{aligned}
{\left[\begin{array}{rr}
\hat{Y}-U T_{i} & -U \\
\hat{X}+V T_{i} & V
\end{array}\right] } \\
=\left[\begin{array}{rr}
0 & I \\
-I & 0
\end{array}\right]\left[\begin{array}{rr}
V & -\hat{X} \\
U & \hat{Y}
\end{array}\right]\left[\begin{array}{rr}
-T_{i} & -I \\
I & 0
\end{array}\right] .
\end{aligned}
$$

According to the stable finiteness of $\mathscr{S}$ and (4), $L_{i}$ admits a strong right representation $\left[\begin{array}{c}\hat{Y}-U T_{i} \\ \hat{X}+V T_{i}\end{array}\right]$ with $\left[\begin{array}{rr}\hat{Y}-U T_{i} & -U \\ \hat{X}+V T_{i} & V\end{array}\right]$ invertible in $\mathscr{S}^{2 \times 2}$. Since

$$
C_{0} \hat{X}+C_{0} V T_{1}+\hat{Y}-U T_{1}=\left(I+C_{0} L_{1}\right)\left(\hat{Y}-U T_{1}\right)
$$

is invertible in $\mathscr{L}$, the operator

$$
R=\left(C_{0} \hat{X}+C_{0} V T_{1}+\hat{Y}-U T_{1}\right)^{-1}\left(C_{0} V-U\right)
$$

is well-defined. Let $\left[-\hat{N}_{C_{0}} \hat{M}_{C_{0}}\right]$ be a strong left representation of $C_{0}$. It follows from the stability of $\left\{L_{1}, C_{0}\right\}$ that

$$
\begin{aligned}
R=\left\{\hat{M}_{C_{0}}\left(\hat{X}+V T_{1}\right)+\right. & \left.\hat{N}_{C_{0}}\left(\hat{Y}-U T_{1}\right)\right\}^{-1} \\
& \times\left(\hat{N}_{C_{0}} V-\hat{M}_{C_{0}} U\right) \in \mathscr{S} .
\end{aligned}
$$

Observe that

$$
\begin{aligned}
V & -\hat{X} R-V T_{1} R \\
& =V-\left(\hat{X}+V T_{1}\right)\left(C_{0} \hat{X}+C_{0} V T_{1}+\hat{Y}-U T_{1}\right)^{-1}\left(C_{0} V-U\right) \\
& =V-L_{1}\left(I+C_{0} L_{1}\right)^{-1} C_{0} V+L_{1}\left(I+C_{0} L_{1}\right)^{-1} U \\
& =\left(I+L_{1} C_{0}\right)^{-1}\left(I+L_{1} C_{2}\right) V .
\end{aligned}
$$

Hence $V-\hat{X} R-V T_{1} R$ is invertible in $\mathscr{L}$. It follows from (6) that

$$
C_{0}=\left(U+\hat{Y} R-U T_{1} R\right)\left(V-\hat{X} R-V T_{1} R\right)^{-1} .
$$

Again applying the stable finiteness of $\mathscr{S}$,

$$
\begin{aligned}
& {\left[\begin{array}{cc}
V-\hat{X} R-V T_{1} R & -\left(\hat{X}+V T_{1}\right) \\
U+\hat{Y} R-U T_{1} R & \hat{Y}-U T_{1}
\end{array}\right]} \\
& \quad=\left[\begin{array}{rr}
0 & I \\
-I & 0
\end{array}\right]\left[\begin{array}{cr}
\hat{Y}-U T_{1} & -U \\
\hat{X}+V T_{1} & V
\end{array}\right]\left[\begin{array}{rr}
-R & -I \\
I & 0
\end{array}\right]
\end{aligned}
$$

is invertible in $\mathscr{S}^{2 \times 2}$. Therefore, $\left[\begin{array}{l}V-\hat{X} R-V T_{1} R \\ U+\hat{Y} R-U T_{1} R\end{array}\right]$ is a strong right representation of $C_{0}$. According to Lemma 3 and Lemma $4, L_{0}, L_{1}, L_{2}$ are simultaneously stabilized by a linear system $C$ if and only if $C$ has a strong right representation $\left[\begin{array}{l}V-\left(\hat{X}+V T_{1}\right) Q \\ U+\left(\hat{Y}-U T_{1}\right) Q\end{array}\right]$, where $Q \in \mathscr{S}$ satisfies

$$
\hat{M}_{L_{i}}\left(V-\left(\hat{X}+V T_{1}\right) Q\right)+\hat{N}_{L_{i}}\left(U+\left(\hat{Y}-U T_{1}\right) Q\right)
$$

being invertible in $\mathscr{S}$ for $i=0$, 2. Since $\hat{M}_{L_{2}} V+\hat{N}_{L_{2}} U$ is invertible in $\mathscr{S}$, for $i=2$, (10) is invertible in $\mathscr{S}$ if and only if

$$
\begin{aligned}
\left(\hat{M}_{L_{2}} V+\hat{N}_{L_{2}} U\right)^{-1}\left[\hat{M}_{L_{2}} V-\hat{M}_{L_{2}}\left(\hat{X}+V T_{1}\right) Q+\hat{N}_{L_{2}} U+\hat{N}_{L_{2}}\left(\hat{Y}-U T_{1}\right) Q\right] & \\
= & I+\left(\hat{M}_{L_{2}} V+\hat{N}_{L_{2}} U\right)^{-1}\left(\hat{N}_{L_{2}}-\hat{M}_{L_{2}} L_{1}\right)\left(\hat{Y}-U T_{1}\right) Q \\
& =I+\left(V+L_{2} U\right)^{-1}\left(L_{2}-L_{1}\right)\left(\hat{Y}-U T_{1}\right) Q
\end{aligned}
$$


is invertible in $\mathscr{S}$. Since $C_{0}$ stabilizes $L_{0}$, we have

$$
\begin{aligned}
\hat{M}_{L_{0}}\left(V-\hat{X} R-V T_{1} R\right)+\hat{N}_{L_{0}}\left(U+\hat{Y} R-U T_{1} R\right) & \hat{M}_{L_{0}}\left(L_{0} U+V+\left(L_{0}-L_{1}\right)\left(\hat{Y}-U T_{1}\right) R\right) \\
= & \hat{M}_{L_{0}}\left[L_{0} U+V+\left(L_{0}-L_{1}\right)\left(\hat{Y}-U T_{1}\right)\right. \\
& \left.\quad \times\left(C_{0} \hat{X}+C_{0} V T_{1}+\hat{Y}-U T_{1}\right)^{-1}\left(C_{0} V-U\right)\right] \\
= & \hat{M}_{L_{0}}\left(L_{0} U+V+\left(L_{0}-L_{1}\right)\left(I+C_{0} L_{1}\right)^{-1}\left(C_{0} V-U\right)\right)
\end{aligned}
$$

is invertible in $\mathscr{S}$. Then, for $i=0,(10)$ is invertible in $\mathscr{S}$ if and only if

$$
\begin{aligned}
& {\left[\hat{M}_{L_{0}}\left(V-\left(\hat{X}+V T_{1}\right) R\right)+\hat{N}_{L_{0}}\left(U+\left(\hat{Y}-U T_{1}\right) R\right)\right]^{-1}} \\
& \times\left[\hat{M}_{L_{0}} V-\hat{M}_{L_{0}}\left(\hat{X}+V T_{1}\right) Q+\hat{N}_{L_{0}} U+\hat{N}_{L_{0}}\left(\hat{Y}-U T_{1}\right) Q\right] \\
& =\left[L_{0} U+V+\left(L_{0}-L_{1}\right)\left(I+C_{0} L_{1}\right)^{-1}\left(C_{0} V-U\right)\right]^{-1} \\
& \times\left[L_{0} U+V+\left(L_{0}-L_{1}\right)\left(\hat{Y}-U T_{1}\right) Q\right]
\end{aligned}
$$

is invertible in $\mathscr{S}$. The proof is complete.

As a consequence, we give a sufficient condition for the simultaneous stabilizability of three plants. The criteria listed in the following are much simpler than those given in Theorem 1 in Ref. 14.

Corollary 1 Suppose that $C_{0}$ stabilizes $L_{0}$ and $L_{1}$ and $C_{2}$ stabilizes $L_{1}$ and $L_{2}$. Let $[V U]^{\mathrm{T}}$ be a strong right representation of $C_{2}$. If

$$
\begin{aligned}
& \|\left(L_{1} U+V\right)^{-1}\left(I+L_{1} C_{0}\right)\left(I+L_{0} C_{0}\right)^{-1} \\
& \quad \times\left(L_{0}-L_{1}\right)\left(I+C_{0} L_{1}\right)^{-1}\left(C_{0} V-U\right) \|<1,
\end{aligned}
$$

then $L_{0}, L_{1}$, and $L_{2}$ are stabilizable.

Proof: Observe that

$$
\begin{aligned}
\Delta= & L_{0} U+V+\left(L_{0}-L_{1}\right)\left(I+C_{0} L_{1}\right)^{-1}\left(C_{0} V-U\right) \\
= & L_{0} U+L_{0}\left(I+C_{0} L_{1}\right)^{-1} C_{0} V+\left(I+L_{1} C_{0}\right)^{-1} V \\
& -L_{0}\left(I+C_{0} L_{1}\right)^{-1} U+L_{1}\left(I+C_{0} L_{1}\right)^{-1} U \\
= & L_{0} C_{0}\left(I+L_{1} C_{0}\right)^{-1} L_{1} U+L_{0} C_{0}\left(I+L_{1} C_{0}\right)^{-1} V \\
& +\left(I+L_{1} C_{0}\right)^{-1} V+\left(I+L_{1} C_{0}\right)^{-1} L_{1} U \\
= & \left(I+L_{0} C_{0}\right)\left(I+L_{1} C_{0}\right)^{-1}\left(L_{1} U+V\right) .
\end{aligned}
$$

Therefore, (1) becomes

$\Delta^{-1}\left[\Delta-\left(L_{0}-L_{1}\right)\left(I+C_{0} L_{1}\right)^{-1}\left(C_{0} V-U\right)+\left(L_{0}-L_{1}\right)\left(\hat{Y}-U T_{1}\right) Q\right]$ $=I-\Delta^{-1}\left(L_{0}-L_{1}\right)\left(I+C_{0} L_{1}\right)^{-1}\left(C_{0} V-U\right)+\Delta^{-1}\left(L_{0}-L_{1}\right)\left(\hat{Y}-U T_{1}\right) Q$

Since $\left\|\Delta^{-1}\left(L_{0}-L_{1}\right)\left(I+C_{0} L_{1}\right)^{-1}\left(C_{0} V-U\right)\right\|<1$ from (11), we have $\Delta^{-1}\left(L_{0}-L_{1}\right)\left(I+C_{0} L_{1}\right)^{-1}\left(C_{0} V-U\right) \in$ $\mathscr{S}$. Then

$$
\begin{aligned}
& \left(I-\Delta^{-1}\left(L_{0}-L_{1}\right)\left(I+C_{0} L_{1}\right)^{-1}\left(C_{0} V-U\right)\right)^{-1} \\
& =\sum_{n=0}^{\infty}\left(\Delta^{-1}\left(L_{0}-L_{1}\right)\left(I+C_{0} L_{1}\right)^{-1}\left(C_{0} V-U\right)\right)^{n} \in \mathscr{S},
\end{aligned}
$$

and then one can choose $Q=0$ such that (1) and (2) are both invertible in $\mathscr{S}$.

Remark 2 If one of the stabilizing controllers in transmission condition is stable, the simultaneous stabilizability criteria can be characterized without any information about strong representations.

Corollary 2 Suppose that $C_{0} \in \mathscr{L}$ stabilizes $L_{0}$ and $L_{1}$, and $C_{2} \in \mathscr{S}$ stabilizes $L_{1}$ and $L_{2}$. Then a linear system $C \in \mathscr{L}$ simultaneously stabilizes $L_{0}, L_{1}, L_{2}$ if and only if $C$ has a strong right representation $\left[\begin{array}{c}I-\left(I+C_{2} L_{1}\right)^{-1} Q \\ C_{2}+L_{1}\left(I+C_{2} L_{1}\right)^{-1} Q\end{array}\right]$, where $Q \in \mathscr{S}$ such that

$$
\begin{aligned}
\left(I+L_{1} C_{2}\right)^{-1}\left(I+L_{1} C_{0}\right)\left(I+L_{0} C_{0}\right)^{-1} & \\
\times & \times\left[I+L_{0} C_{2}+\left(L_{0}-L_{1}\right)\left(I+C_{2} L_{1}\right)^{-1} Q\right]
\end{aligned}
$$

and

$$
I+\left(I+L_{2} C_{2}\right)^{-1}\left(L_{2}-L_{1}\right)\left(I+C_{2} L_{1}\right)^{-1} Q
$$

are both invertible in $\mathscr{S}$.

Proof: If $C_{2} \in \mathscr{S}$, then $C_{2}$ has a strong right representation $\left[I C_{2}\right]^{\mathrm{T}}$ with $\left[\begin{array}{cc}I & 0 \\ C_{2} & I\end{array}\right]$ invertible in $\mathscr{S}^{2 \times 2}$. The result is clear by Theorem 1 .

\section{EXAMPLE}

In this section we present an example to demonstrate the effectiveness and applicability of the proposed method in Theorem 1. Consider the following three plants,

$$
L_{0}=\left[\begin{array}{ccccccc}
-1 & & & & & & \\
a_{1} & 2 & & & & & \\
a_{2} & b_{2} & \frac{8}{3} & & & & \\
a_{3} & b_{3} & 0 & \frac{15}{4} & & & \\
\vdots & \vdots & \vdots & \ddots & \ddots & & \\
a_{n} & b_{n} & 0 & \cdots & 0 & \frac{n^{2}+2 n}{n+1} & \\
\vdots & \vdots & \vdots & \cdots & \vdots & \ddots & \ddots
\end{array}\right],
$$

$L_{1}=I$, and

$$
L_{2}=\left[\begin{array}{rrrrrrrr}
\frac{1}{2} & & & & & & \\
-1 & 2 & & & & & \\
2^{2} & -2^{3} & 4 & & & & \\
-2^{3} & 2^{4} & -2^{3} & 2 & & & \\
2^{5} & -2^{6} & 2^{5} & -2^{3} & 4 & & \\
-2^{6} & 2^{7} & -2^{6} & 2^{4} & -2^{3} & 2 & \\
\vdots & \vdots & \vdots & \vdots & \vdots & \ldots & \ddots
\end{array}\right]
$$


where $\left\{a_{n}: n \geqslant 1\right\}$ and $\left\{b_{n}: n \geqslant 2\right\}$ are two bounded sequences in $\mathbb{C}$. Note that the computation for the strong representation of $L_{0}$ is complicated, in fact there is no explicit method to compute the strong representation of general infinitedimensional lower-triangular operator. However, it is easy to check that $C_{0}=\operatorname{diag}\{0,0,3,4, \ldots\}$ is a common stabilizer for $L_{0}, L_{1}$, and $C_{2}=I$ simultaneously stabilizes $L_{1}, L_{2}$. Clearly, $\left[I C_{2}\right]^{\mathrm{T}}$ is a strong right representation of $C_{2}$ with $\left[\begin{array}{cc}I & 0 \\ C_{2} & I\end{array}\right]$ invertible in $\mathscr{S}^{2 \times 2}$. That is, the parameters in Theorem 1 are chosen to be $U=V=\hat{Y}=I$ and $\hat{X}=0$. We compute that

$$
\begin{aligned}
& L_{0} U+V+\left(L_{0}-L_{1}\right)\left(I+C_{0} L_{1}\right)^{-1}\left(C_{0} V-U\right) \\
& =L_{0}+I+\left(L_{0}-I\right)\left(I+C_{0}\right)^{-1}\left(C_{0}-C_{2}\right) \\
& =\operatorname{diag}\left\{2,2, \frac{9}{2}, \frac{32}{5}, \cdots, \frac{2 n^{2}+4 n+2}{n+2}, \ldots\right\},
\end{aligned}
$$

then

$$
\begin{aligned}
{\left[L_{0} U\right.} & \left.+V+\left(L_{0}-L_{1}\right)\left(I+C_{0} L_{1}\right)^{-1}\left(C_{0} V-U\right)\right]^{-1} \\
& \times\left[L_{0} U+V+\left(L_{0}-L_{1}\right)\left(\hat{Y}-U T_{1}\right) Q\right] \\
& =\left[\begin{array}{ccccccc}
0 & & & & & \\
\frac{a_{1}}{2} & \frac{3}{2} & & & & \\
\frac{2 a_{2}}{9} & \frac{2 b_{2}}{9} & \frac{2 \cdot 11}{3 \cdot 9} & & & \\
\frac{5 a_{3}}{32} & \frac{5 b_{3}}{32} & 0 & \frac{5 \cdot 19}{4 \cdot 32} & & \\
\vdots & \vdots & \vdots & \ddots & \ddots & \\
\frac{(n+2) a_{n}}{2 n^{2}+4 n+2} & \frac{(n+2) b_{n}}{2 n^{2}+4 n+2} & 0 & \ldots & 0 & \ddots
\end{array}\right] \\
& +\left[\begin{array}{cccccc}
\frac{1}{2} & & & & & \\
\frac{a_{1}}{4} & \frac{1}{4} & & & & \\
\frac{a_{2}}{9} & \frac{b_{2}}{9} & \frac{5}{27} & & & \\
\frac{5 a_{3}}{64} & \frac{5 b_{3}}{64} & 0 & \frac{5 \cdot 11}{64 \cdot 4} & & \\
\vdots & \vdots & \vdots & \ddots & \ddots & \\
\frac{(n+2) a_{n}}{2\left(2 n^{2}+4 n+2\right)} & \frac{(n+2) b_{n}}{2\left(2 n^{2}+4 n+2\right)} & 0 & \cdots & 0 & \ddots
\end{array}\right]
\end{aligned}
$$

Also, we compute

$$
\begin{aligned}
& I+\left(V+L_{2} U\right)^{-1}\left(L_{2}-L_{1}\right)\left(\hat{Y}-U T_{1}\right) Q \\
& =I+\left(I+L_{2}\right)^{-1}\left(L_{2}-I\right)\left(I+C_{2} L_{1}\right)^{-1} Q \\
& =I+\frac{1}{2}\left(I+L_{2}\right)^{-1}\left(L_{2}-I\right) Q \\
& =I+\left[\begin{array}{ccccc}
-\frac{1}{6} & & & & \\
-\frac{2}{3^{2}} & \frac{1}{6} & & & \\
\frac{2}{3} \frac{4}{5} & -\frac{2}{3} \frac{4}{5} & \frac{3}{10} & & \\
-\frac{2^{2}}{3^{3}} \frac{4}{5} & \frac{2^{2}}{3^{2}} \frac{4}{5} & -\frac{2}{3} \frac{4}{5} & \frac{1}{6} & \\
\frac{2^{2}}{3^{3}} 4^{2} & -\frac{2^{2}}{3^{2}} 4^{2} & \frac{2}{3} 4^{2} & -\frac{2}{3} \frac{4}{5} & \frac{3}{10} \\
-\frac{2^{3}}{3^{2}} \frac{4}{2}^{2} & \frac{2}{3}^{3} \frac{4}{2}^{2} & -\frac{2^{2}}{3^{2}} \frac{4}{2}^{2} & \frac{2^{2}}{3^{2}} \frac{4}{5} & -\frac{2}{3} \frac{4}{5} \\
\vdots & \vdots & \vdots & \vdots & \vdots
\end{array}\right.
\end{aligned}
$$

Choosing $Q=\operatorname{diag}\{-2,0,0,0, \ldots\}$ gives

$$
\begin{aligned}
I+\left(V+L_{2} U\right)^{-1}\left(L_{2}-L_{1}\right)\left(\hat{Y}-U T_{1}\right) Q \\
=\left[\begin{array}{cccccccc}
\frac{4}{3} & & & & & & \\
\frac{2^{2}}{3^{2}} & 1 & & & & & \\
-\frac{2^{2}}{3^{2}} \frac{4}{5} & 0 & 1 & & & & \\
\frac{2^{3}}{3^{3}} \frac{4}{5} & 0 & 0 & 1 & & & \\
-\frac{2^{3}}{3^{3}} \frac{4^{2}}{5^{2}} & 0 & 0 & 0 & 1 & & \\
\vdots & \vdots & \vdots & \vdots & \ddots & \ddots & \\
c_{n} & 0 & 0 & 0 & \cdots & 0 & 1
\end{array}\right],
\end{aligned}
$$

where $C_{n}=\left\{\begin{aligned}-\left(\frac{2}{3}\right)^{k+1}\left(\frac{4}{5}\right)^{k}, & n=2 k, \\ \left(\frac{2}{3}\right)^{k+2}\left(\frac{4}{5}\right)^{k}, & n=2 k+1 .\end{aligned}\right.$

Since $\lim _{n \rightarrow \infty} C_{n}=0$, we have $I+\left(V+L_{2} U\right)^{-1}\left(L_{2}-\right.$ $\left.L_{1}\right)\left(\hat{Y}-U T_{1}\right) Q$ invertible in $\mathscr{S}$. Note that

$$
\begin{aligned}
& \left(L_{0} U+V+\left(L_{0}-L_{1}\right)\left(I+C_{0} L_{1}\right)^{-1}\left(C_{0} V-U\right)\right)^{-1} \\
& \times\left(L_{0} U+V+\left(L_{0}-L_{1}\right)\left(\hat{Y}-U T_{1}\right) Q\right) \\
& =\left[\begin{array}{cccccc}
1 & & & & & \\
0 & \frac{3}{2} & & & & \\
0 & \frac{2 b_{2}}{9} & \frac{2 \cdot 11}{3 \cdot 9} & & & \\
0 & \frac{5 b_{3}}{64} & 0 & \frac{5 \cdot 19}{4 \cdot 32} & & \\
0 & \vdots & 0 & \ddots & \ddots & \\
0 & \frac{(n+2) b_{n}}{2\left(2 n^{2}+4 n+2\right)} & 0 & \ldots & 0 & \frac{(n+2)\left(n^{2}+3 n+1\right)}{(n+1)\left(2 n^{2}+4 n+2\right)} \\
\vdots & \vdots & \vdots & \ldots & \vdots & \vdots
\end{array}\right] .
\end{aligned}
$$

Since the sequence $\left\{b_{n}: n \geqslant 2\right\}$ is bounded, we have $\lim _{n \rightarrow \infty}(n+2) b_{n} / 2\left(2 n^{2}+4 n+2\right)=0$. It is clear that any diagonal element of the above matrix is nonzero and

$$
\lim _{n \rightarrow \infty} \frac{(n+2)\left(n^{2}+3 n+1\right)}{(n+1)\left(2 n^{2}+4 n+2\right)}=\frac{1}{2},
$$

then

$$
\begin{aligned}
\left(L_{0} U+V+\left(L_{0}\right.\right. & \left.\left.-L_{1}\right)\left(I+C_{0} L_{1}\right)^{-1}\left(C_{0} V-U\right)\right)^{-1} \\
& \times\left(L_{0} U+V+\left(L_{0}-L_{1}\right)\left(\hat{Y}-U T_{1}\right) Q\right)
\end{aligned}
$$

is invertible in $\mathscr{S}$.

According to Theorem 1 , we conclude that $L_{0}$, $L_{1}$, and $L_{2}$ can be simultaneously stabilized. Again applying Theorem 1 , we can construct a simultaneous stabilizer for $L_{0}, L_{1}$, and $L_{2}$ as

$$
\begin{aligned}
C & =\left(U+\left(\hat{X}+V T_{1}\right) Q\right)\left(V-\left(\hat{Y}-U T_{1}\right) Q\right)^{-1} \\
& =\left(C_{2}+L_{1}\left(I+C_{2} L_{1}\right)^{-1} Q\right)\left(I-\left(I+C_{2} L_{1}\right)^{-1} Q\right)^{-1} \\
& =\left(I+\frac{Q}{2}\right)\left(I-\frac{Q}{2}\right)^{-1} \\
& =\operatorname{diag}\left\{\frac{1}{2}, 1,1,1,1, \ldots\right\} .
\end{aligned}
$$


Since the parameters $a_{n}$ and $b_{n}$ of $L_{0}$ are irregular, the computation of strong representation for $L_{0}$ is complicated. The previous work in Refs. 14, 15 has demonstrated their applications. Compared with the criteria in Theorem 1, the design method of simultaneous stabilizing controllers in Theorem 1 really is quite simple when the strong representation of a controller in transmission condition is clear.

\section{CONCLUSION}

This paper has dealt with the simultaneous stabilization problem of three discrete time-varying systems from the transitivity version. This simultaneous stabilizability criteria extends the results in Refs. 14, 15 by requiring only the strong representation of a controller in the transmission condition. It is noted that the main results can cover all the discretetime time-varying linear system with the realization described by the difference equations

$$
\begin{aligned}
x_{n+1} & =a_{n} x_{n}+b_{n} u_{n}, \quad x_{0}=0, \\
y_{n} & =c_{n} x_{n}+d_{n} u_{n},
\end{aligned}
$$

where $x_{n}, u_{n}, y_{n} \in \mathbb{C}^{k}$ are the state, the input, and the output of the plant, $a_{n}, b_{n}, c_{n}, d_{n}$ are complex matrices of appropriate dimensions. Clearly, the time-varying linear system is the operator from $u=\left(u_{0}, u_{1}, \ldots, u_{n}, \ldots\right)$ to $y=\left(y_{0}, y_{1}, \ldots, y_{n}, \ldots\right)$ with matrix representation $\left[\begin{array}{ccccc}d_{0} & & & & \\ c_{1} b_{0} & d_{1} & & & \\ c_{2} a_{1} b_{0} & c_{2} b_{1} & d_{2} & & \\ c_{3} a_{2} a_{1} b_{0} & c_{3} a_{2} b_{1} & c_{3} b_{2} & d_{3} & \\ \vdots & \vdots & \vdots & \vdots & \ddots\end{array}\right]$.

Acknowledgements: This research is supported by NSFC (No. 11871131, 11671065) and the Fundamental Research Funds for the Central Universities (NO. DUT18LK17).

\section{REFERENCES}

1. Zhou B (2016) On asymptotic stability of linear timevarying systems. Automatica 68, 266-76.

2. Zhou B, Zhao T (2017) On asymptotic stability of discrete-time linear time-varying systems. IEEE Trans Automat Contr 62, 4274-81.
3. Chen G, Yang Y (2016) New stability conditions for a class of linear time varying systems. Automatica 71, 342-7.

4. Zhou B, Luo WW (2018) Improved Razumikhin and Krasovskii stability criteria for time-varying stochastic time-delay systems. Automatica 91, 256-69.

5. Feintuch A (2012) On the strong stabilization of slowly time-varying linear systems. Systems Control Lett 61, 112-6.

6. Cantoni M, Jonsson U, Khong SZ (2013) Robust stability analysis for feedback interconnections of time-varying linear systems. SIAM J Control Optim 51, 353-79.

7. Liu L, Lu YF (2014) Stabilizability, representations and factorizations for the time-varying linear system. System Control Lett 66, 58-64.

8. Saeks R, Murray J (1982) Fractional representation algebraic geometry, and the simultaneous stabilization problem. IEEE Trans Automat Contr 27, 895-903.

9. Vidyasagar M, Viswanadham N (1982) Algebraic design techniques for reliable stabilization. IEEE Trans Automat Contr 27, 1085-95.

10. Lu YF, Xu XP (2008) The stabilization problem for discrete time-varying linear systems. Systems Control Lett 57, 936-9.

11. Feintuch A (1998) Robust Control Theory in Hilbert Space, Springer-Verlag.

12. Yu TQ (2011) The transitivity in simultaneous stabilization. System Control Lett 60, 1-6.

13. Liu L, Lu YF (2013) Necessary and sufficient conditions to the transitivity in simultaneous stabilization of time-varying systems. IET Control Theory A 7, 1834-42.

14. Wang HZ, Yu TQ, Xiao JM (2016) Simultaneously stabilising controllers for time-varying linear systems within the framework of nest algebras. Int J Control 89, 1632-40.

15. Liu L, Lu YF (2015) Transitivity in simultaneous stabilization for a family of time-varying systems. System Control Lett 78, 55-62.

16. Dale WN, Smith MC (1993) Stabilizability and existence of system representations for discrete-time time-varying systems. SIAM J Control Optim 31, 1538-57.

17. Davidson KR, Ji YQ (2009) Topological stable rank of nest algebras. P Lon Math Soc 98, 652-78. 